\title{
Estradiol-mediated tumor neo-vascularization
}

\author{
SHILPI RAJORIA ${ }^{1}$, ROBERT SURIANO ${ }^{1}$, YUSHAN L. WILSON ${ }^{2}$, ANDREA L. GEORGE $^{1}$, \\ JAN GELIEBTER $^{1}$, STIMSON P. SCHANTZ ${ }^{2}$ and RAJ K. TIWARI ${ }^{1}$ \\ ${ }^{1}$ Department of Microbiology and Immunology, New York Medical College, Valhalla, NY 10595; \\ ${ }^{2}$ Department of Otolaryngology, New York Eye and Ear Infirmary, New York, NY 10003, USA
}

Received November 3, 2010; Accepted January 12, 2011

DOI: $10.3892 / \mathrm{ol} .2011 .283$

\begin{abstract}
Neo-vascularization is essential for tumor growth and metastasis and is presumably initiated by bone marrowderived endothelial progenitor cells (BM-EPCs). These cells predominantly reside in the bone marrow and are recruited at sites of inflammation, tissue damage and tumors. The tissuespecific factors responsible for recruitment of BM-EPCs and neo-vascularization are the subject of intense investigation. Using bone marrow cells from Tek/green fluorescent protein (GFP) transgenic mice, we analyzed the effect of estrogen on the mobilization of BM-EPCs to orthotopically implanted cancer cells in estrogen- and non-estrogen-supplemented ovariectomized mice. The donor marrow cells were unique as they were fluorescently tagged, allowing for the tracking of their migration to the tumor tissues. Results showed that GFP + BM-EPCs were incorporated within the tumor vasculature in comparison to the sham injections. Notably, estrogen supplementation enhanced the mobilization of BM-EPCs to the tumor site. This elevation shows that estrogen may affect tumor neo-vascularization by inducing the mobilization of BM-EPCs. Understanding and characterizing the mechanism involved in the estrogen-induced mobilization of BM-EPCs may serve as a 'Trojan horse' in the delivery of bio-molecules that may disrupt tumor vasculogenesis and induce the targeted killing of tumor cells.
\end{abstract}

\section{Introduction}

Estrogens are steroid hormones that play a crucial role in the growth, differentiation and function of sexual and reproductive organs. Estrogens are produced naturally by the body as three biochemically distinct hormones, estrone, $17 \beta$-estradiol and estriol, with $17 \beta$-estradiol being the key and most potent estrogen in human tissues. Estrogens are metabolized to 2-hydroxyestrone (2-OHE1) and 16-ahydroxyestrone

Correspondence to: Dr Raj K. Tiwari, Department of Microbiology and Immunology, New York Medical College, Valhalla, NY 10595, USA

E-mail: raj_tiwari@nymc.edu

Key words: estrogen, bone marrow-endothelial progenitor cells, neo-vascularization
(16-OHE1), and their relative concentrations in the female body, based on their estrogen agonist 16-OHE1 or antagonist 2-OHE1 activity, were reported to increase a female individual's risk for breast, uterine and other hormonally induced cancers (1). The cellular activity of estrogen is known to be mediated by its interaction with its receptors, either estrogen receptor (ER)- $\alpha$ or ER- $\beta$, resulting in an induction of the growth regulator signal transduction pathway (2-4). Estrogen mediates cell proliferation via the genomic pathway by inducing the transcription of various genes, such as c-jun, c-myc and c-fos, and growth factors, as well as having a direct impact on cyclins that regulate the cell cycle (5). Furthermore, the non-genomic pathways cause the binding of estrogen to either membrane-bound estrogen receptor, resulting in the activation of a number of intracellular signaling pathways, such as PI3K/Akt and ERK, which upon activation lead to anti-apoptotic signals (6-8). Malignant transformation of cells may lead to the dysregulation of ER signaling, resulting in evasion of apoptosis, self-induced growth signals and clonogenicity, and ultimately malignant transformation $(2,6)$.

Apart from epithelial cell proliferation and growth, estrogen also modulates endothelial cell growth, tubulogenesis and angiogenesis (9). Angiogenesis is an indispensable process for tumor growth and metastasis involving the sprouting of new blood vessels from pre-existing capillaries (9). On the other hand, neo-vascularization involves the generation of new blood vessels from endothelial progenitor cells. Bone marrow-derived endothelial progenitor cells (BM-EPCs) which normally reside in the bone marrow are significant mediators of neo-vascularization (10). BM-EPCs have the potential of proliferating, mobilizing and differentiating into mature endothelial cells in response to pro-angiogenic factors, such as vascular endothelial growth factor (VEGF) and matrix metalloproteinases (MMPs) (10-15). Under normal conditions, the generation of new vessels is regulated by a complex interaction network of activators and inhibitors of angiogenesis. However, in case of inflammation, injury or cancer, this balance between pro- and anti-angiogenic factors is tilted towards the pro-angiogenic factors. This leads to mobilization, homing and incorporation of BM-EPCs at the diseased site, remodeling of the extracellular matrix and anastomoses of surrounding pre-existing and new vessels, resulting in neo-vascularization (10-15). Recently, estrogen was found to act as a mobilizing agent for breast cancer-responsive neo-vascularization (16). 
Increased angiogenesis, a major process crucial for the development of new blood vessels, was extensively observed in thyroid proliferative disease including Graves' disease, hyperplastic goiter and thyroid cancer (17). Over 200 million people worldwide are affected by thyroid proliferative disease (TPD), which includes hypothyroidism, hyperthyroidism, adenoma, goiter and cancer, and their incidences have been on the increase, particularly among women (18). Notably, according to the American Thyroid Association, one in every eight female individuals are likely to develop some type of thyroid disorder in their lifetime (19). Observational studies indicate that pregnancy, oral contraceptives and estrogen replacement therapy increase the risk of TPD with a decrease in the risk of thyroid malignancies following menopause (20). This marked gender bias warrants investigation into the factors, most obviously estrogen, that make 'being female' such a high risk factor for TPD. A recent study from our laboratory, as well as studies from the literature provide evidence that thyroid cells express the functional estrogen receptor and are estrogen-responsive (21-23). Estrogen potentially modulates the metastasis of thyroid cancer cells, although the role of estrogen in thyroid neo-vascularization remains to be determined (23). Based on the past literature and our findings, the present study was designed to examine the contribution of estrogen in BM-EPC mobilization towards implanted transformed cells using the well-characterized Tie2/green fluorescent protein (GFP) in vivo model. In this model, BM-EPCs express GFP under the transcriptional control of endothelial cell-specific tyrosine kinase promoter Tek. It was found that estrogen mobilized BM-EPCs to the tumor site and mediated neo-vascularization in an in vivo-based experimental system.

\section{Materials and methods}

Cell culture. KAT50-TS cells were provided by Dr Kenneth B. Ain (VA Medical Center, Lexington, KY, USA). This cell line has been misidentified as being of thyroid origin (24). However, DNA profiling using the Identifier kit from Applied Biosystems (data not shown) confirmed that HT-29(50-TS) is not of thyroid cancer origin but matches the short tandem repeat (STR) profile of the HT-29 colorectal cancer cell line. Thus, by our own convention, the cell line name HT-29(50-TS) was designated. This name identifies the parent cell line, but distinguishes the two cell lines as unique sublines that may exhibit differential responses compared to similar treatments of HT-29 in another laboratory. Irrespective of the origin, the cancer cells grow well in thyroid tissue, and our experimental results remain validated. Cells were cultured in phenol red-free RPMI-1640 (Mediatech, Herndon, VA, USA) supplemented with $10 \%$ fetal bovine serum (FBS; Atlanta Biologicals, Lawrenceville, GA, USA), penicillin 10,000 IU/ml, streptomycin 10,000 $\mu \mathrm{g} / \mathrm{ml}$ (Mediatech), $2 \mathrm{mM}$ L-glutamine (Mediatech), $100 \mathrm{mM}$ MEM sodium pyruvate solution (Mediatech) and $10 \mathrm{mM}$ MEM non-essential amino acid solution (Mediatech).

Western blot analysis. Human tumor cells were routinely maintained in culture and harvested using trypsin, and then washed with phophate-buffered saline (PBS) twice. Cytoplasmic and nuclear lysates were prepared using the NE-PER nuclear and cytoplasmic extraction reagent kit by Pierce (Rockford, IL, USA). The cytoplasmic and nuclear fractions were separated according to the manufacturer's instructions. Western blot analysis was then performed for anti-ER- $\alpha$ and anti-ER- $\beta$ antibodies (Santa Cruz Biotechnology, Santa Cruz, CA, USA).

Trypan blue experiment. Cells $\left(1 \times 10^{5}\right)$ were plated in complete medium in 6-well culture dishes and were allowed to adhere overnight. The following day the cells were washed with PBS, starved using serum- and phenol red-free medium, 10\% charcoal stripped FBS (Sigma Chemical Co., St. Louis, MO, USA) and penicillin $10,000 \mathrm{IU} / \mathrm{ml}$ for $24 \mathrm{~h}$ or left in $10 \% \mathrm{FBS}$ as the control. Subsequently, cells were treated with $10^{-8} \mathrm{M}$ estradiol $\left(\mathrm{E}_{2}\right)$ (Sigma Chemical Co.) or left untreated as the control. After $24 \mathrm{~h}$, the cells were harvested and stained using $0.4 \%$ trypan blue solution (Sigma Chemical Co.). The number of viable (unstained) and dead (stained) cells was counted using a hemocytometer, and the stimulation of cell growth by $\mathrm{E}_{2}$ was calculated as the increase in viable cell count for cells treated with $\mathrm{E}_{2}$ relative to the control cells.

Animals. Female BALBc/nu/nu mice (aged 8-12 weeks) were purchased from Charles River Laboratories International, Inc. (Wilmington, MA, USA). The mice were housed and maintained in laminar flow cabinets under specific pathogen-free conditions in facilities approved by the American Association of Laboratory Animal Care in accordance with current regulations and standards of the U.S. Department of Agriculture, the U.S. Department of Health and Human Services, the New York State Department of Health, and the NIH. The mice were used in accordance with the Animal Care and Use Guidelines of New York Medical College (Valhalla, NY, USA) under a protocol approved by the Institutional Animal Care Use Committee (no. 149-2-1007).

Experimental model. Mice were grouped as follows: non-ovariectomized (intact), ovariectomized (OVX) and ovariectomized + estrogen-supplemented $\left(\mathrm{OVX}+\mathrm{E}_{2}\right)$. Estrogen supplementation was administered by implantation of a 90-day release pellet containing $1.7 \mathrm{mg} 17 \beta$-estradiol (Innovative Research of America, Sarasota, FL, USA). Seven days later, human cancer cells $\left(100 \times 10^{6}\right.$ cells per $\left.\mathrm{ml}\right)$ were injected in $5 \mu \mathrm{l}$ aliquots into the right thyroid lobe of the OVX and $\mathrm{OVX}+\mathrm{E}_{2}$ ) mouse. On the other hand, control mice received $5 \mu \mathrm{l}$ of PBS as a sham injection. One week following the human cancer cell injections, bone marrow obtained from the Tek/ GFP transgenic mice was harvested, and bone marrow cells ( $4 \times 10^{6}$ cells in $0.1 \mathrm{ml}$ of saline) were transplanted via tail vein injection to all of the mice. The donor marrow cells are unique in that they were populated by BM-EPCs expressing GFP under the transcriptional control of endothelial cell-specific promoter Tek (formerly Tie2). Since these endothelial cells are GFP-tagged, their migration from the marrow to tissues were tracked. No animals met the criteria for sacrifice prior to the end of the treatment period. After a total of two weeks following the tumor cell injections, the mice were sacrificed, and the cervical trachea along with the normal contralateral thyroid gland and thyroid tumors were harvested and mounted into frozen sections prepared for light microscopy and immunofluorescence microscopy. 
A

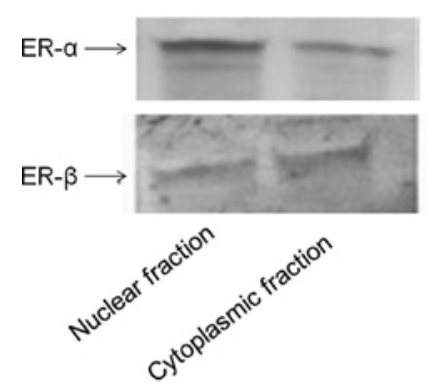

B

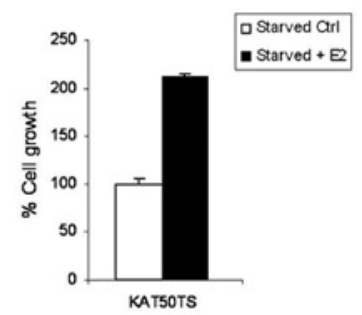

Figure 1. The tumor cells expressed the estrogen receptors and were estrogenresponsive. (A) The nuclear and cytoplasmic protein fractions (15 $\mu \mathrm{g})$ were resolved by SDS-PAGE. Western blot analysis was performed for ER- $\alpha$ and ER- $\beta$. (B) The effect of estradiol on proliferative activity was determined using trypan blue dye exclusion assay. Cell growth was expressed as a percentage, calculated as the increase in the viable cell count for cells treated with estradiol (black bars) relative to the untreated cells (white bars).

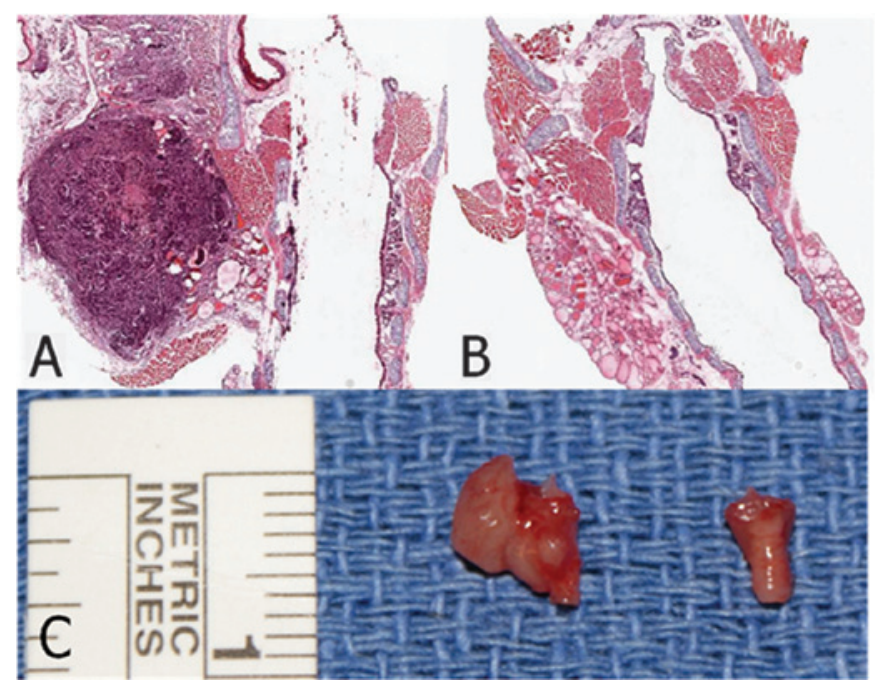

Figure 2. Hematoxylin and eosin staining of (A) implanted human cancer cell tumors in nude mice compared to (B) the controls receiving sham injections with PBS. (C) Gross depictions of the harvested tumor and control specimens.

Immunofluorescent analysis. Capillaries were identified as tubular structures positive for isolectin B4. BM-EPCs were identified as attached spindle-shaped cells, double-positive for GFP and isolectin B4. The number of double-positive cells in 15 fields under $\times 20$ magnification was counted per sample in a blinded manner.

\section{Results}

Estrogen modulates its effects at cellular levels via estrogen receptors. As an initial step in the investigation of the estrogenmediated tumor responsiveness of BM-EPCs, we ascertained the presence of estrogen receptors in the human tumor cells used in the present study. Western blot analysis of the nuclear and cytoplasmic extracts of the implanted tumor cells revealed that the two isoforms of estrogen receptors, ER- $\alpha$ and ER- $\beta$, were present in the cell line (Fig. 1A). The presence of estrogen receptors was also validated by their responsiveness to estradiol-mediated enhancement of proliferation (Fig. 1B).
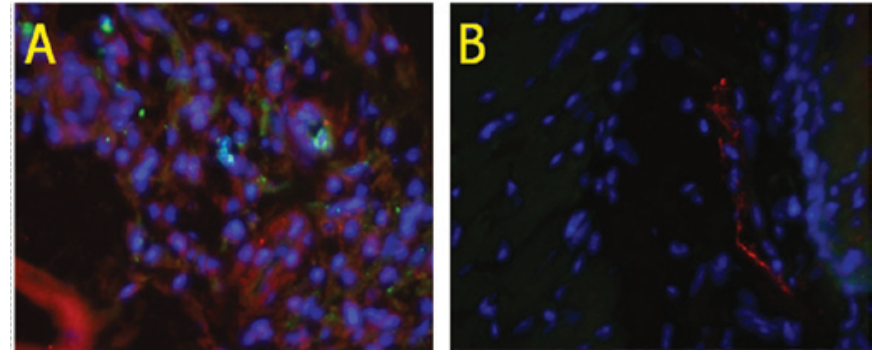

Figure 3. BM-EPCs mobilize to the tumor tissue. Enhanced numbers of injected BM-EPCs (green) present in the (A) tumor bed as compared to the controls receiving (B) sham injections. Red, isolectin B4; blue, DAPI.
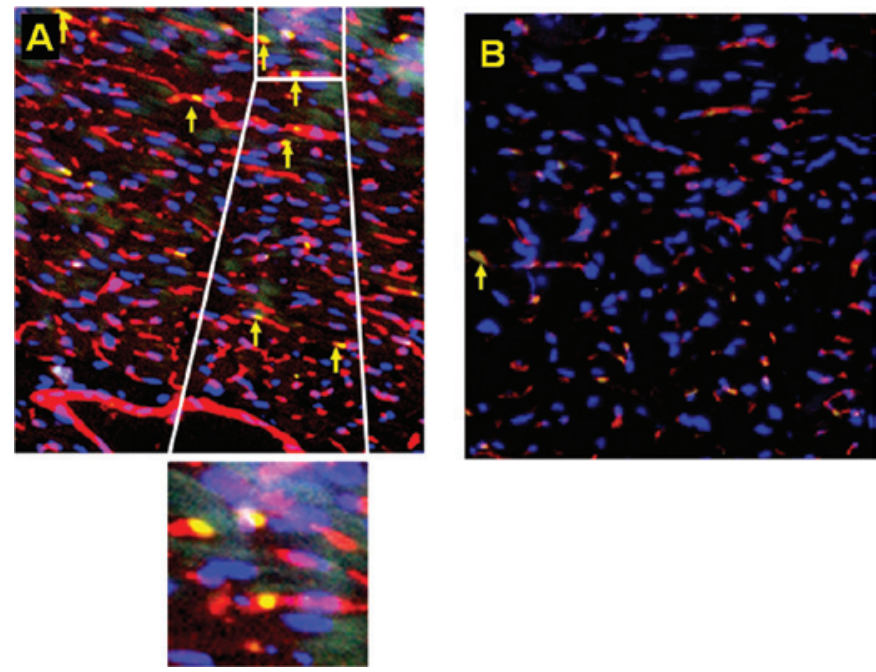

Figure 4. BM-EPCs migrate to the tumor tissue. Various GFP+BM-EPCs physically integrated into the tumor neo-vasculature (A) showing a merged image of isolectin B4 (vasculature, red) and GFP (green) double-positive vessels. Magnification of the inset indicates numerous isolectin B4/GFP double-positive vessels within the tumor bed. (B) For sham injections very few Tie2-GFP+EPCs homed to the tumor tissue. Red, isolectin B4; blue, DAPI; yellow, isolectin B4+GFP double-positive cells.
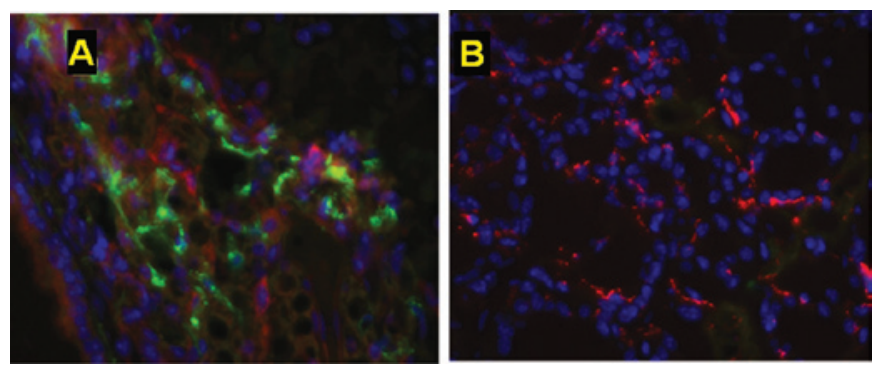

Figure 5. Estrogen enhances BM-EPC mobilization to the tumor tissue Increased numbers of BM-EPCs mobilized to the estrogen-supplemented mouse tumor bed (A: $+\mathrm{E}_{2}+$ tumor cells) as compared to the controls receiving sham injections (B: $+\mathrm{E}_{2}$ - tumor cells) or non-estrogen-supplemented tumor-injected mice (Fig. 3A, -E2 + tumor cells). Red, isolectin B4; blue, DAPI

Previous studies implicated estrogen in the onset of angiogenesis and neo-vascularization in estrogen-responsive tissues, such as breast, including a study from our laboratory. Results of our previous study showed that estradiol mobilizes breast 
cancer-responsive BM-EPCs, initiating neo-vascularization. In the present study, the ability of estrogen to mobilize BM-EPCs towards implanted human cancer cells in the thyroid gland was investigated. Ovariectomized female BALB/c nude mice were supplemented with or without estradiol pellets. After 7 days of estradiol supplementation, mice were injected with human cancer cells, and at day 14, tail vein injections of BM-EPCs isolated from Tek/GFP transgenic mice were administered. Mice were sacrificed after 21 days, and tumors were harvested and analyzed by light microscopy and immunofluorescence microscopy. The implantation of $5 \times 10^{5}$ cancer cells/mouse resulted in $100 \%$ tumor incidence for all groups of mice, with the histology of the tumors being consistent among the groups (Fig. 2).

$\mathrm{Tek}^{+} \mathrm{GFP}^{+}$cells were counted as a representative population of BM-EPCs. Since these cells were also GFP-tagged, their migratory pattern in response to tumor growth and estradiol were easily traced. Capillaries were identified as tubular structures positive for isolectin B4, a marker for progenitor cells of endothelial origin. Tumor xenografts exhibited more BM-EPC mobilization in comparison to the sham-injected group, suggesting that the tumors secreted various paracrine factors that mobilized tumor-responsive BM-EPCS (Fig. 3). Furthermore, increased numbers of BM-EPCs were incorporated into the tumor neo-vasculature compared to the sham-injected group, suggesting that not only do EPCs home to the tumor but physically associate to the blood vessels (Fig. 4). This homing of the EPCs to the tumor tissue was considerably enhanced in the estrogen-supplemented mice compared to the non-estrogen-supplemented tumor-injected group (Fig. 5). In contrast, in the absence of estrogen supplementation or tumor inoculation, few injected BM-EPCs mobilized to the tumor tissue (Fig. 3B). These data indicate that the use of estradiol resulted in an enhanced recruitment of BM-derived cells to the neo-vasculature of the tumor gland.

\section{Discussion}

Angiogenesis is a critical event for normal growth and development as well as malignant growth and metastasis. The process occurs throughout our lifespan beginning as early as embryogenesis and is ongoing throughout postnatal life. Previous studies found that the mechanism of embryogenic angiogenesis is different from that which occurs in adults, commonly termed as postnatal vasculogenesis and neo-vascularization (13). Neo-vascularization is a process noted in the revascularization of ischemic tissue and wound healing, as well as a number of diseases, such as diabetic retinopathy and more recently in various types of cancer (25). Endothelial progenitor cells, which are mainly located in the bone marrow niche postnatally, play a crucial role in the process of neo-vascularization. These cells are phenotypically characterized as $\mathrm{CD}^{4} 4^{+}, \mathrm{CD} 133^{+}$ and VEGF receptor $2^{+}$(VEGFR-2 ${ }^{+}$, also known as KDR or Flk1) (26). The process involves the release of endothelial progenitor cells from the bone marrow to the blood circulation in response to various signals, including stimuli produced by cancer cells, followed by homing to the source, and in the case of tumor signals, to the tumor bed. Progenitor stem cells then differentiate into mature endothelial cells, assisting in ongoing vascular development and angiogenesis.
Various endogenous and exogenous factors have the potential to mobilize EPCs from the bone marrow to blood circulation. Growth factors, cytokines, colony-stimulating factor, VEGF and GM-CSF are significant endogenous factors that mobilize EPCs and promote angiogenesis as well as neovascularization (27). Cholesterol-lowering drugs [HMG-COA reductase inhibitors (statins)] and the peroxisome proliferatoractivated receptor- $\gamma$ (PPAR- $\gamma$ ) agonist are major exogenous factors that enhance the mobilization of EPCs and induce angiogenesis (28). Extreme physical exercise is also reported to increase EPC mobilization and vascular functions in humans (28). Counterattacking these pro-angiogenic factors and blocking the mobilization of BM-EPCs inhibits the growth and metastasis of tumors. This process indicates a strong potential for cancer treatment and has been identified as a potential target for antitumor therapies. Recent therapeutic trials have attempted to harness this potential for neo-vascularization into treatment protocols $(29,30)$.

It is believed that the cancer-promoting properties of estrogen stem from the effects of various pro-survival pathways that are activated by the binding of estrogen to its receptor. The correlation of estrogen exposure to the thyroid gland and its contribution to TPD is lacking. There is an obvious gender bias in thyroid cancer, alluding to the putative role of estrogen. Our data aimed to clarify this relationship by utilizing a body of evidence indicating BM-EPCs as significant factors involved in neo-vascularization. In this context, our data revealed that BM-EPCs homed to xenograft tumors inducing neo-vascularization in our in vivo model. Furthermore, this tumor-responsive homing of BM-EPCs to the tumor site was greatly enhanced by estradiol by potentially secreting endothelial and tumor-enhancing factors. Considering that thyroid vascularization is seminal in thyroid proliferative disease, our findings may have clinical utility in using BM-EPCs as a potential 'Trojan horse' by which to deliver bio-molecules that disrupt tumor vasculogenesis and induce the targeted killing of tumor cells.

\section{Acknowledgements}

This study was supported by grants from the National Cancer Institute 1R01CA131946 and the Department of Otolaryngology, New York Eye and Ear Infirmary, New York, NY. The authors gratefully acknowledge the contributions of Dr Raj Kishore (Northwestern University) for his assistance in the immunofluorescent analysis and Dr Jeffrey N. Myers and Dr Maria Gule (The University of Texas M. D. Anderson Cancer Center) for their aid in refining the protocol for injections in the thyroid.

\section{References}

1. Lord RS, Bongiovanni B and Bralley JA: Estrogen metabolism and the diet-cancer connection: rationale for assessing the ratio of urinary hydroxylated estrogen metabolites. Altern Med Rev 7: 112-129, 2002.

2. He YY, Cai B, Yang YX, Liu XL and Wan XP: Estrogenic G protein-coupled receptor 30 signaling is involved in regulation of endometrial carcinoma by promoting proliferation, invasion potential, and interleukin-6 secretion via the MEK/ERK mitogenactivated protein kinase pathway. Cancer Sci 100: 1051-1061, 2009. 
3. Vijayanathan V, Venkiteswaran S, Nair SK, Verma A, Thomas TJ Zhu BT and Thomas T: Physiologic levels of 2-methoxyestradiol interfere with nongenomic signaling of $17 \beta$-estradiol in human breast cancer cells. Clin Cancer Res 12: 2038-2048, 2006.

4. Lewis-Wambi JS and Jordan VC: Estrogen regulation of apoptosis: how can one hormone stimulate and inhibit? Breast Cancer Res 11: 206, 2009.

5. Ciocca DR and Fanelli MA: Estrogen receptors and cell proliferation in breast cancer. Trends Endocrinol Metab 8: 313-321, 1997.

6. Song RX and Santen RJ: Membrane initiated estrogen signaling in breast cancer. Biol Reprod 75: 9-16, 2006.

7. Hua K, Feng W, Cao Q, Zhou X, Lu X and Feng Y: Estrogen and progestin regulate metastasis through the PI3K/AKT pathway in human ovarian cancer. Int J Oncol 33: 959-967, 2008.

8. Pietras RJ: Interaction between estrogen and growth factor receptors in human breast cancers and tumor-associated vasculature. Breast J 9: 361-373, 2003.

9. Losordo DW and Isner JM: Estrogen and angiogenesis: A review. Arterioscler Thromb Vasc Biol 21: 6-12, 2001

10. Risau W: Mechanism of angiogenesis. Nature 386: 671-674, 1997.

11. Aghi M and Chiocca EA: Contribution of bone marrow-derived cells to blood vessels in ischemic tissues and tumors. Mol Ther 6: 994-1005, 2005.

12. Young PP, Vaughan DE and Hatzopoulos AK: Biological properties of endothelial progenitor cells (EPCs) and their potential for cell therapy. Prog Cardiovasc Dis 49: 421-429, 2007.

13. Masuda $\mathrm{H}$ and Asahara T: Post-natal endothelial progenitor cells for neovascularization in tissue regeneration. Cardiovasc Res 58 390-398, 2003.

14. Rehman J, Li J, Orschell CM and March KL: Peripheral blood 'endothelial progenitor cells' are derived from monocytes/ macrophages and secrete angiogenic growth factors. Circulation 107: 1164-1169, 2003.

15. Asahara T, Takahashi T, Masuda H, Kalka C, Chen D, Iwaguro $\mathrm{H}$, Inai $\mathrm{Y}$, Silver $\mathrm{M}$ and Isner JM: VEGF contributes to postnatal neovascularization by mobilizing bone marrowderived endothelial progenitor cells. EMBO J 18: 3964-3972, 1999.

16. Suriano R, Chaudhuri D, Johnson RS, Lambers E, Ashok BT, Kishore $\mathrm{R}$ and Tiwari RK: 17 $\beta$-Estradiol mobilizes bone marrow-derived endothelial progenitor cells to tumors. Cancer Res 68: 6038-6042, 2008

17. Ramsden JD: Angiogenesis in the thyroid gland. J Endocrinol 166 : 475-480, 2000
18. Jemal A, Siegel R, Ward E, Hao Y, Xu J and Thun MJ: Cancer statistics. CA Cancer J Clin 59: 225-249, 2009.

19. Cook MB, Dawsey SM, Freedman ND, Inskip PD, Wichner SM, Quraishi SM, Devesa SS and McGlynn KA: Sex disparities in cancer incidence by period and age. Cancer Epidemiol Biomarkers Prev 18: 1174-1182, 2009.

20. Levi F, Franceschi S, Gulie C, Negri E and Vecchia CL: Female thyroid cancer: the role of reproductive and hormonal factors in Switzerland. Oncology 50: 309-315, 1993.

21. Furlanetto TW, Nguyen LQ and Jameson JL: Estradiol increases proliferation and down-regulates the sodium/iodide symporter gene in FRTL-5 cells. Endocrinology 140: 5705-5711, 1999.

22. Manole D, Schildknecht B, Gosnell B, Adams E and Derwahl M: Estrogen promotes growth of human thyroid tumor cells by different molecular mechanisms. J Clin Endocrinol Metab 86: 1072-1077, 2001.

23. Rajoria S, Suriano R, Shanmugam A, Wilson YL, Schantz SP, Geliebter J and Tiwari RK: Metastatic phenotype is regulated by estrogen in thyroid cells. Thyroid 20: 33-41, 2010.

24. Schweppe RE, Klopper JP, Korch C, Pugazhenthi U, Benezra M, Knauf JA, Fagin JA, Marlow L, Copland JA, Smallridge RC and Haugen BR: Deoxyribonucleic acid profiling analysis of 40 human thyroid cancer cell lines reveals cross-contamination resulting in cell line redundancy and misidentification. J Clin Endocrinol Metab 93: 4331-4341, 2008.

25. Asahara T, Masuda H, Takahashi T, Kalka C, Patore C, Silver M, Marianne K, Magner M and Isner JM: Bone marrow origin of endothelial progenitor cells responsible for postnatal vasculogenesis in physiological and pathological neovascularization. Circ Res 85: 221-228, 1999.

26. Gehling UM, Ergun S, Schumacher U, Wagener C, Pantel K, Otte M, Schuch G, Schafhausen P, Mende T, Kilic N, Kluge K, Schafer B, Hossfeld DK and Fiedler W: In vitro differentiation of endothelial cells from AC133-positive progenitor cells. Blood 95: 3106-3112, 2000.

27. Khakhoo AY and Finkel T: Endothelial progenitor cell. Ann Rev Med 56: 79-101, 2005.

28. Dong F and Ha X-Q: Effect of endothelial progenitor cells in neovascularization and their application in tumor therapy. China Med J 123: 2454-2460, 2010.

29. Wu LC and Zhang WD: Clinical trials of antiangiogenesis therapy on gastric cancer. Gastroenterol Res 1: 14-19, 2008

30. Kerbel RS: Antiangiogenic therapy: A universal chemosensitization strategy for cancer? Science 312: 1171-1175, 2006. 